\title{
Underlying topological Dirac nodal line mechanism of anomalously large electron-phonon coupling strength on Be (0001) surface
}

\author{
Ronghan $\mathrm{Li}^{1,2}$, Jiangxu $\mathrm{Li}^{1,2}$, Lei Wang ${ }^{1,2}$, Hui $\mathrm{Ma}^{1}$, Dianzhong $\mathrm{Li}^{1}$, Yiyi $\mathrm{Li}^{1}$, and Xing-Qiu Chen ${ }^{1 *}$ \\ ${ }^{1}$ Shenyang National Laboratory for Materials Science, Institute of Metal Research, \\ Chinese Academy of Science, 110016 Shenyang, Liaoning, China and \\ ${ }^{2}$ School of Materials Science and Engineering, University of Science and Technology of China, Heifei, P. R. China
}

(Dated: July 22, 2019)

\begin{abstract}
Beryllium was recently discovered to harbor a Dirac nodal line (DNL) in its bulk phase and the DNL-induced non-trivial drumhead-like surface states (DNSSs) on its (0001) surface, rationalizing several already-existing historic puzzles [Phys. Rev. Lett., 117, 096401 (2016)]. However, to date the underlying mechanism, as to why its (0001) surface exhibits an anomalously large electron-phonon coupling effect $\left(\lambda_{e-p h}^{s} \approx 1.0\right)$, remains unresolved. Here, by means of first-principles calculations we have evidenced that the coupling of the DNSSs with the phononic states mainly contributes to its novel surface $e$-ph enhancement. Besides that the experimentally observed $\lambda_{e-p h}^{s}$ and the main Eliashberg coupling function (ECF) peaks have been reproduced well, we have decomposed the ECF, $\alpha^{2} F(k, \boldsymbol{q} ; v)$, and the $e$-ph coupling strength $\lambda(k, \boldsymbol{q} ; v)$ as a function of each electron momentum $(k)$, each phonon momentum $(\boldsymbol{q})$ and each phonon mode $(v)$, evidencing the robust connection between the DNSSs and both $\alpha^{2} F(k, \boldsymbol{q} ; v)$ and $\lambda(k, \boldsymbol{q} ; v)$. The results reveal the strong $e$-ph coupling between the DNSSs and the phonon modes, which contributes over $80 \%$ of the $\lambda_{e-p h}^{s}$ coefficient on the Be (0001) surface. It highlights that the anomalously large $e$-ph coefficient on the Be (0001) surface can be attributed to the presence of its DNL-induced DNSSs, clarifying the long-term debated mechanism.
\end{abstract}

In difference from both topological Dirac semimetals and topological Weyl semimetals which host isolated Dirac cones and Weyl nodes in their bulk phases, the class of topological Dirac nodal line semimetals (DNLs) [1-57] exhibit the fully closed lines around the Fermi level due to the continuously linear crossings of the bulk energy bands in the lattice momentum space. The projection of the DNLs onto a certain surface would result in a closed ring, in which the topologically protected nearly-flat drumhead-like non-trivial surface states (DNSSs) occur. This kind of exotic band structures render various novel properties. Besides the common DNSSs-induced high electronic density around the Fermi energy on the surface, there are still giant surface Friedel oscillation [5], flat Landau level [56], long-range Coulomb interaction [57], special collective modes [25], flat optical conductivity [29-32], giant magnetoresistance and mobility [28], and unconventional enhancement of effective mass [33] as well as a potential route to achieve high-temperature superconductivity [22-24] and catalytic candidates [47], and so on.

Recently, the discoveries of the DNL in the pure beryllium metal [5] and this DNL-induced robust DNSSs on its (0001) surface rationalize three already-existing historic puzzles [5865] of $(i)$ the long-standing question of the surface states observed by the angle-resolved photoemission (ARPES) experiments, (ii) the underlying physics of the severe deviations of its surface electronic structures from the description of the nearly free electron picture, and (iii) the substantial mechanism of the giant Friedel oscillations on the (0001) surface. Although these three puzzles have been resolved, the origin of its anomalously large electron-phonon (e-ph) coupling effect on the (0001) surface still remains opening. As early as in the 1998, the e-ph coupling strength, $\lambda_{e-p h}^{s}$, on the Be

*Corresponding author: xingqiu.chen@imr.ac.cn
(0001) surface was measured to be $1.15 \pm 0.1$ or $1.18 \pm 0.07$ by the ARPES experiment [66, 67], being about five times the bulk Be value $\lambda_{e-p h}^{b}=0.24[69,70]$. On basis of these experimental measurements, the authors claimed that the surface superconductivity with a potential high critical temperature may exist on the Be (0001) surface [66]. The subsequent ARPES reports on the $e$-ph coupling strength $\lambda_{e-p h}^{s}$ was in a range from 0.6 to 1.18 [66-68, 71, 72, 75, 76], mainly because of the anisotropic $e$-ph interaction on the $\mathrm{Be}(0001) \bar{\Gamma}$ surface states [77]. However, the reported low $\lambda_{e-p h}^{s}=0.6$ [68] was experimentally found to be indeed caused by surface oxygen contamination [77]. Although ab initio calculations for Be surface reproduced both the measured geometric and electronic structures as well as surface phonon dispersions, the known calculations for the $e$-ph coupling on Be (0001) surface did not reproduce the measurements $[77,78]$. The early DFT-derived $\lambda_{e-p h}^{s}$ is 0.90 [72, 73], but the subsequent work in Ref. [77] stated that the previously calculated value was wrong due to the programming error. The latest ARPES experiments [78] even claimed that none of the main experimental Eliashberg coupling function (ECF) peaks in the low-frequency range was captured by the previous calculations $[72,73]$. Therefore, the mechanism dramatically triggering the anomalously large $e$-ph coupling strength of the $\mathrm{Be}(0001)$ surface remains opening and the discrepancies between experimental measurement and theoretical calculations would also need to be urgently clarified.

Returning to the fact that bulk Be exhibits the novel DNL and the DNL-induced DNSSs on its (0001) surface [5], we have strongly suspected whether or not the highly localized and nearly flat DNSSs result in the occurrence of the anomalously large e-ph coupling strength. Here, through first-principles calculations within the framework of Density Function Theory (DFT) $[79,80]$ by employing the Quantum Espresso [81] (QE) code with the norm-conserving pseudopotential [82](details 
refer to supplementary method [83]), we have revisited the problem of the $e-p h$ coupling strength of the $\mathrm{Be}(0001)$ surface by establishing the connection of both the ECF, $\alpha^{2} F(k, \boldsymbol{q} ; v)$, and the $e$-ph coupling strength, $\lambda(k, \boldsymbol{q} ; v)$. Remarkably, we have evidenced the strong $e-p h$ coupling of the DNSSs with the phonon modes, contributing over $80 \%$ of the $\lambda_{e-p h}^{s}$ of the $\mathrm{Be}(0001)$ surface. Accordingly, these results confirm that the anomalously large $e$-ph coupling strength on the Be (0001) surface is substantially ascribed to the topologically protected DNL-induced DNSSs.

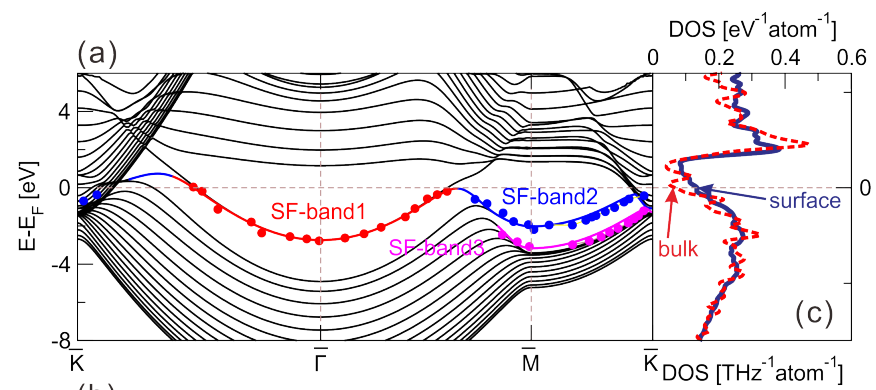

(b)

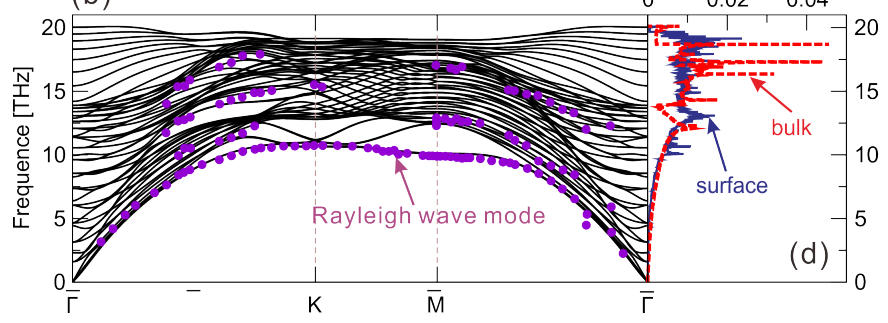

Figure 1: DFT-derived electronic band structure $(\mathrm{a}, \mathrm{c})$ and phonon dispersion (b,d) of the (0001) surface in the hcp Be metal. Panel (a): the surface electronic band structures along the high-symmetric lines as compared with available ARPES experimental data. Panel (b): the surface phonon dispersion along the high-symmetric lines as compared with available experimentally observed surface phonon dispersions. Panel (c and d): the surface electronic (c) and phonon (d) densities of states in comparison with the total density of states of its bulk phase.

The current calculations of the electronic band structures have reproduced well our previously published results [5]. The DNL projection onto the (0001) surface exhibits a closed ring surrounding $\bar{\Gamma}$ in which the topologically protected DNSSs appear [5]. Along the $\bar{K}-\bar{\Gamma}-\bar{M}$ paths in the surface BZ, these DNSSs [see SF-band1 in Fig. 1(a)] disperse parabolically around the centered $\bar{\Gamma}$ point, in nice agreement with the experimental findings obtained by ARPES [58,60]. In addition, we have derived the surface phonon dispersions, as shown in Fig. 1(b and d). Our calculations are in a nice agreement with the experimentally observed dispersions $[58,59,61,68,71,77]$ and the previous calculations [72,74]. Particularly, it can be seen that the highly localized surface phonon mode [Reyleigh waves (RW) mode [76]] in Fig. 2(b), dominated by the vibration of the topmost atom along the surface normal, is soft and very sensitive to the interplanar spacing between the first and second topmost atomic layers. In comparison with its bulk phase, the Be (0001) surface shows the apparent differences: (i) the surface electronic density at the Fermi level is much larger than that of the bulk phase due to the presence of the DNSSs (Fig. 1c), and (ii) the two extra peaks at $10 \mathrm{THz}$ and 11 $\mathrm{THz}$ occur in the surface phonon density and the apparent large peak is shift to a higher frequency of $12.5 \mathrm{THz}$ with respect to that of its bulk phase (Fig. 1d).

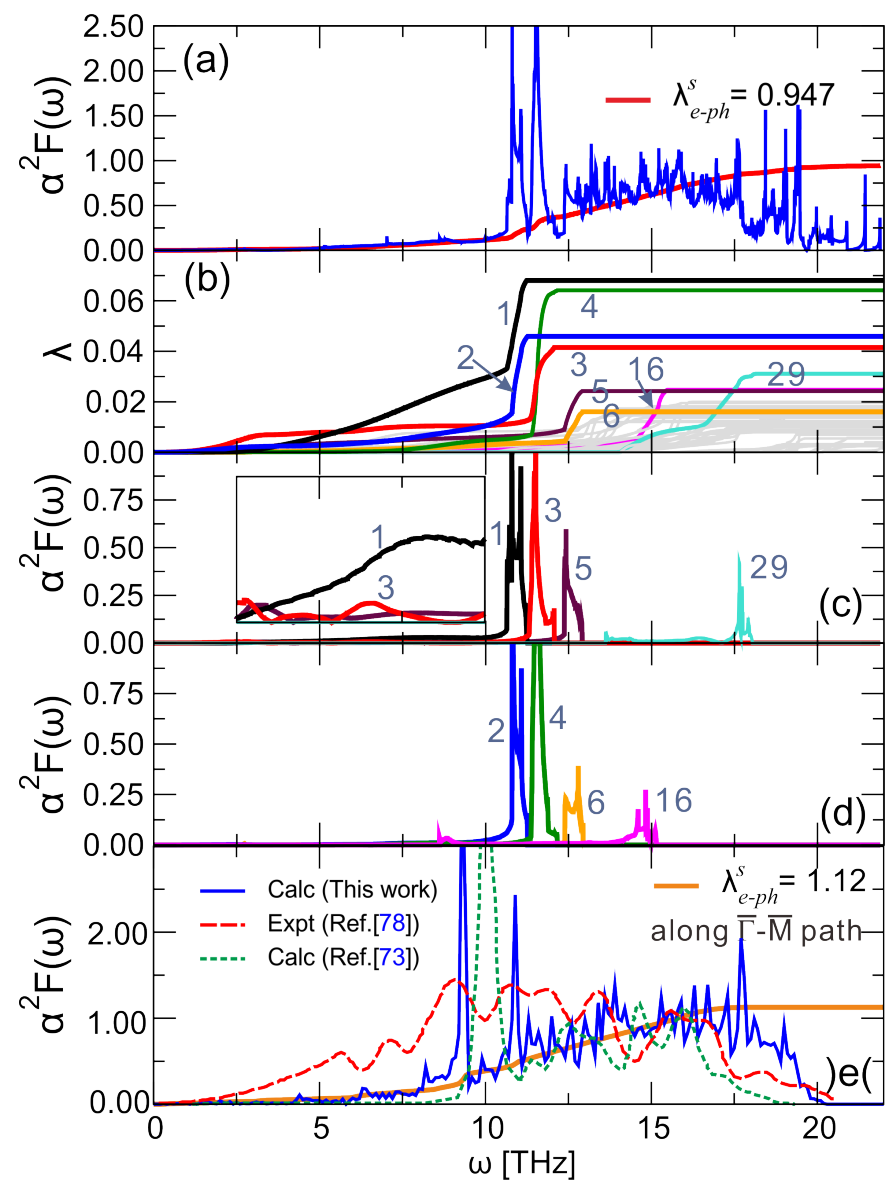

Figure 2: DFT-derived Eliashberg coupling function (ECF), $\alpha^{2} F(\omega)$, and the coupling strength, $\lambda$, of the Be (0001) surface. Panel (a): the total ECF and the total $e$ - $p h$ coupling strength; Panel (b): 48 moderesolved $e$ - $p h$ coupling strengths, $\lambda$. The $\lambda$ values of No. $1 \sim$ No.6, No.16 and No.29 modes are highlighted and the other 40 modes are plot in a grey background; Panel (c and d): the mode-resolved ECFs for eight selected phonon modes [No.1 (RW mode), No.3, No.5 and No.29 modes in panel (c); No.2, No.4, No.6 and No.16 modes in panel (d)]; Panel (e): the derived ECF along the $\bar{\Gamma}-\bar{M}$ direction as compared with the ARPES-extracted data multiplied with a factor of 3 for easy comparison [78] and previous calculated data [73].

We have theoretically derived the ECF, $\alpha^{2} F(\omega)$, and the $e$-ph coupling strength, $\lambda$, for both the bulk phase and the (0001) surface [see supplementary Fig. S1 [83] and Fig. 2]. The obtained $\lambda_{e-p h}^{b}=0.254$ for the bulk phase (supplementary Fig. S1 [83]) and $\lambda_{e-p h}^{s}=0.947$ for the Be (0001) surface [Fig. 2(a)], in the nice agreement with the experimental data $(0.24$ for bulk phase [69, 70]; $1.15 \pm 0.1$ [66] and $1.18 \pm 0.07$ [67] for the Be (0001) surface). The previous calculations [72, 73] did not correctly reproduced the the frequency ranges of the experimental ECF peaks $[77,78]$ in the low-frequency region along the specified $\bar{\Gamma}-\bar{M}$ direction in the Be (0001) surface. 
As compared in Fig. 2(e), the conspicuous difference in ECF is the large peak in the previous theoretical calculation [72, 73] at about $10 \mathrm{THz}$, on which the experimental data exhibited a valley [78]. Importantly, along the $\bar{\Gamma}-\bar{M}$ direction our current calculations reproduced well these experimentally observed positions of ECF peaks in Fig. 2(e). Furthermore, in order to elucidate whether the electronic DNSSs have effects on the $e$-ph coupling strength on the $\mathrm{Be}(0001)$ surface, we need to derive the ECF $\alpha^{2} F(\omega)$ at the $k$ momenta where the DNSSs would exist in the surface BZ. Therefore, we have decomposed the total ECF $\alpha^{2} F(\omega)$ [84] into the function of each electron momentum $(k)$, each phonon momentum $(\boldsymbol{q})$ and each phonon mode $(v)$ in the (0001) surface BZ as,

$$
\alpha^{2} F(k, \boldsymbol{q} ; v)=\sum_{i, f}\left|g_{\boldsymbol{q}, v}(k, i, f)\right|^{2} \delta\left(\epsilon_{f}-\epsilon_{i} \mp \omega_{\boldsymbol{q}, v}\right),
$$

where the term of $g_{\boldsymbol{q}, v}(k, i, f)$ is the so-called $e$-ph matrix element, which represents the probability of electron scattering from an initial electron state $i$ with a momentum $\boldsymbol{k}$ to a final electron state $f$ interacted by a phonon with a momentum $\boldsymbol{q}$ and a mode index $v$. This term can be derived as follows,

$$
g_{\boldsymbol{q}, v}(k, i, f)=\sqrt{\frac{\hbar}{2 M \omega_{\boldsymbol{q}, v}}}\left\langle\varphi_{i, k}\left|\delta V_{s c f}^{\boldsymbol{q}, v}\right| \varphi_{f, k \pm \boldsymbol{q}}\right\rangle,
$$

where $M$ is the atom mass and $\delta V_{s c f}^{q, v}$ is the gradient of the self-consistent potential of the atomic displacements induced by the phonon mode $v$ with a momentum $\boldsymbol{q}$ [84]. In addition, the total $e$-ph coupling strength can be decomposed into $\lambda(\boldsymbol{q} ; v)$ of each phonon momentum $(\boldsymbol{q})$ and each phonon mode $(v)$ over all electron $k$ momenta in the (0001) surface BZ as,

$$
\lambda(\boldsymbol{q} ; v)=2 \int \frac{d k}{\Omega_{B Z}} \frac{\alpha^{2} F(k, \boldsymbol{q} ; v)}{N\left(e_{F}\right) \hbar \omega_{\mathbf{q}, v}}
$$

where $N\left(e_{F}\right)$ is the electronic density of states at the Fermi level and $\Omega_{B Z}$ is the area of the surface BZ. In terms of Equ. (3) we have first calculated mode-resolved $\lambda$ over all electron $k$ and phonon $q$ momenta in Fig. 2(b) to elucidate the effects of phonon modes on $\lambda_{e-p h}^{s}$. Among them, the No.1 RW mode contributes the largest $\lambda$ value of 0.068 . In combining both the mode-resolved ECF $\alpha^{2} F(\omega)$ in Fig. 2c and $e$-ph coupling strength, $\lambda$ in Fig. 2b, it can be clearly referred that the $\lambda$ of the soft No.1 RW mode is mainly originated from two parts. The first part is from the broad ECF peak [see the enlarged inset in Fig. 2(c)] in a range from $5 \mathrm{THz} \sim 10 \mathrm{THz}$, in which the ECF along the $\bar{\Gamma}-\bar{M}$ direction makes the great contribution [Fig. 2(e)]. The second part is from the sharp ECF peak in a range of $10.3 \mathrm{THz} \sim 11.2 \mathrm{THz}$ for the No. $1 \mathrm{RW}$ mode. In very similarity to the No. 1 RW mode, the No. 2 mode results in a sharp ECF peak, almost overlapping with the second sharp ECF peak of the No.1 mode in the nearly same frequency region [Fig. 2(a,b,c)]. It also contributes the third largest $\lambda$ value of 0.046 . The second sharp peak in the total ECF in the frequency range from $11.3 \mathrm{THz}$ to $11.9 \mathrm{THz}$ [Fig. 2(a)] is fully comprised by the phonon No.3 and No. 4 modes, which mainly exhibit in-planed vibration of the first topmost atomic layer in coupling with the small out-planar vibration of the second topmost atomic layer along the surface normal. They contribute the fourth and second largest $\lambda$ of 0.044 and 0.064 , respectively. With increasing the frequency to the region from $12.4 \mathrm{THz}$ to $12.9 \mathrm{THz}$ the nearly overlapped ECF peaks occur from both the phonon No.5 and No.6 modes [Fig. 2(b,c,d)], which highlight the atomic vibrations of the first and third topmost atomic layers along the surface normal. They contribute to the relatively large $\lambda$ values of 0.024 and 0.016 , respectively. Both No. 16 and No. 29 modes also contribute a comparably high $\lambda$ of 0.024 and 0.032 in the high-frequency region. Although all other phonon modes contribute a sizable $e$ - $p h$ coupling strength, their contributed $\lambda$ values are in general smaller than those of the above eight modes [Fig. 2(b)]. Therefore, the six modes (No.1 to No.6) in the low-frequency region and the two modes (No.16 and No.29) in the high-frequency region dominantly contribute to the $e$-ph coupling strength. Interestingly, we have even recognized that these eight modes exhibit highly localized surface phonon states [Fig. 1(b)].

In order to understand in-depth the $e$ - $p h$ coupling between the DNSSs and both the ECF and $\lambda_{e-p h}^{s}$, we have derived the $(\boldsymbol{q} ; v)$-resolved $\lambda(\boldsymbol{q} ; v)$ values at each phonon vibration $\boldsymbol{v}$ mode and at each phonon $\boldsymbol{q}$ momentum through Equs. (1 and 3), as compared in Fig. 3(a) and supplementary Fig. $\mathrm{S} 2$ [83]. In a good agreement with previous experimental observations [77, 78], the calculated ECFs at the 2D closed circular $k$ momenta around the centered $\bar{\Gamma}$ are anisotropic [i.e., ECF of No.1 mode in Fig. 3(b)]. We have thus derived $\lambda_{e-p h}^{s}$ along the directions rotating from the $\bar{\Gamma}-\bar{M}$ to $\bar{\Gamma}-\bar{K}$ as shown in supplementary Fig. S3 [83], indicating the apparent anisotropy, again in nice agreement with experimental findings [Fig. 3(c)]. Strikingly, our calculations have evidenced that the DNSSs exhibit the highly strong $e-p h$ coupling with the phonon modes, In particular, at the phonon $\bar{K}$ momentum $\boldsymbol{q}=(1 / 3,1 / 3,0)[85]$ (Fig. 3(a)) their $e$-ph couplings are much stronger than those at all the other 34 phonon $\boldsymbol{q}$ momenta (corresponding to six symmetry-inequivalent $\boldsymbol{q}$ momenta, see supplementary Fig. S3 [83]). In order to clearly visualize how the DNSSs couple with these vibration modes, we have plot the mode-resolved ECFs for nine selected vibration modes in the $k$ momenta of the Be (0001) BZ at $\boldsymbol{q}=(1 / 3,1 / 3,0)$ [85], including the aforementioned eight dominating No.1 No.6, No. 16 and No.29 modes [Fig. 3(d to k)] and the lowest contributed No.31 mode [Fig. 3(1)]. For a sake of the convenient comparison, the electronic Fermi surface is further plot in Fig. 3(b) with the aim of showing the exact $k$ momenta where the DNSSs appear. On the one hand, a closed circle surrounding the centered $\bar{\Gamma}$ point exactly corresponds to the DNSSs, as marked by the SF-band1 in Fig. 1(a) and, on the other hand, three closed triangle-like electronic localized states surrounding the centered $\bar{K}$ point are originated from the topologically trivial surface states [5] [SF-band2 and SF-band3 in Fig. 1(a)]. Remarkably, at each $q$ momentum on the 2D circular $k$ momenta of the Fermi contour where the topological protected DNSSs exactly appear in Fig. 3(c), we have also observed the highest bright closed circular ECFs for all vibration modes, as shown for night selected modes in Fig. 3(e-m). However, at the triangle-like $k$ momenta around $\bar{K}$ where the trivial surface electronic states appear the brightness of the ECFs is much weak than that of the centered 


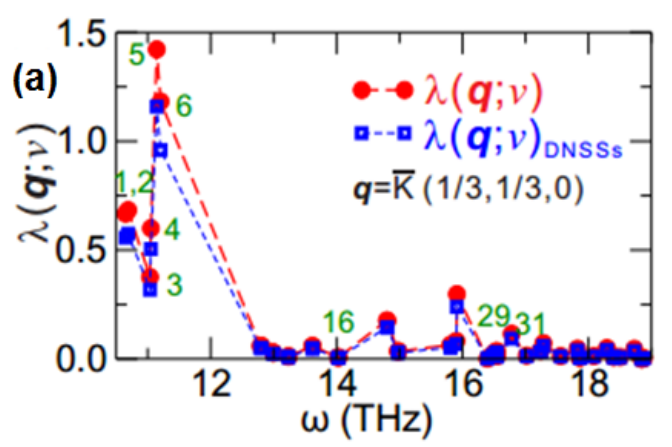

$\begin{array}{ll}\text { (d) Fermi Surf. } & \text { (e) No. } 1\end{array}$

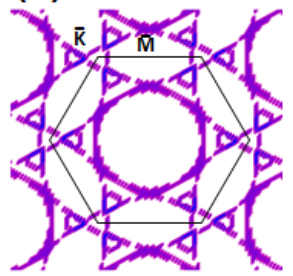

(i) No. 5
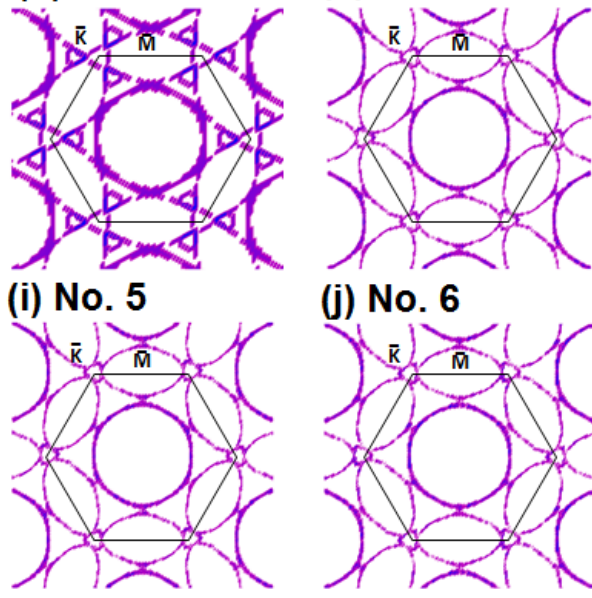

(j) No. 6

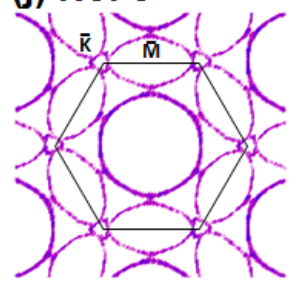

(b)

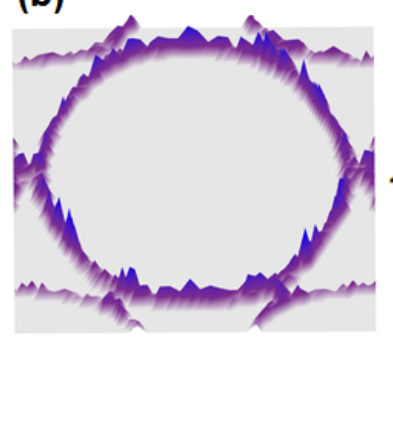

(f) No. 2

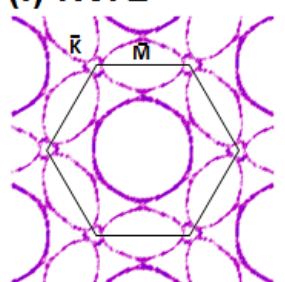

(k) No. 16

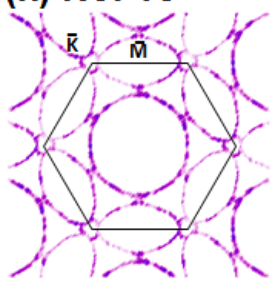

(g) No. 3

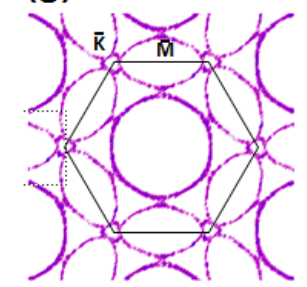

(I) No. 29

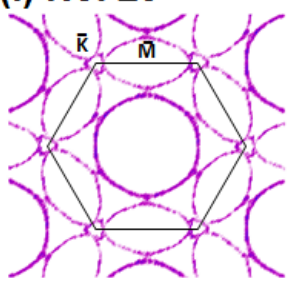

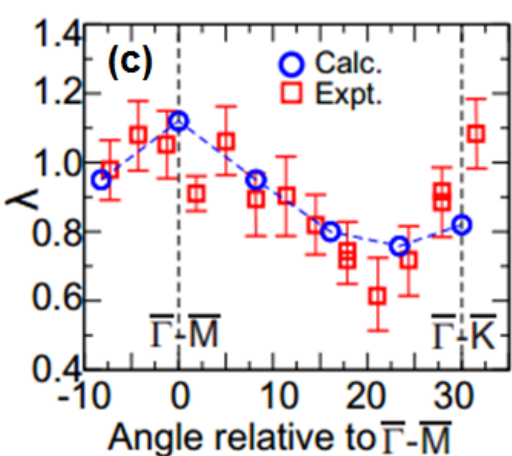

(h) No. 4

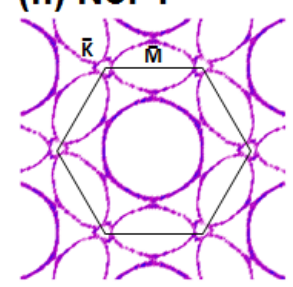

(m) No. 31

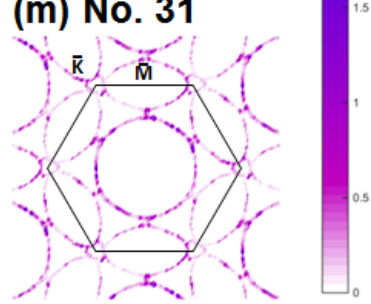

Figure 3: The $(\boldsymbol{q} ; v)$-resolved $\lambda$ and ECFs at the phonon $\bar{K}$ momentum $\boldsymbol{q}=(1 / 3,1 / 3,0)$ [85] on the Be (0001) surface. Panel (a), The (q;v)-resolved $\lambda$ and the number denotes the corresponding vibration mode (the dashed lines are just a guide to the eye). Panel (b), the 3D plot of the ECF of the No.1 mode shows a strong anisotropic $k$-dependent feature. Panel (c), anisotropic $\lambda$ along the directions rotating from the $\bar{\Gamma}-\bar{M}$ to $\bar{\Gamma}-\bar{K}$ by varying the angle relative to the $\bar{\Gamma}-\bar{M}$ direction. Panel (d), The derived electronic Fermi surface of the Be (0001) surface. Panels (e) to (m), the $(\boldsymbol{q} ; v)$-resolved ECFs of No.1 No.6, No.16, No.29, and No.31 modes.

circular ECFs around the $\bar{\Gamma}$. It needs to be emphasized that the $e$-ph couplings are stronger for the eight surface localized phonon modes (No. $1 \sim$ No.6, No. 16 and No. 29 modes). Although the contributions of the other modes to the e-ph coupling are relatively not large, the coupling between the DNSSs and these phonon modes are still obvious. As evidenced in Fig. $3(\mathrm{~m})$ we have visualized the ECFs of the No. 31 mode which exhibits the lowest contribution to $\lambda_{e-p h}^{s}$, the coupled circular ECF can be clearly observed as well. All these facts clearly evidence the robust strong couplings of the DNSSs with the phonon vibration modes on the Be (0001) surface, leading to its anomalously large surface $e$-ph coupling strength.

Furthermore, we have numerically calculated the weight of the $e$-ph coupling between the DNSSs and phonon modes to the total $\lambda_{e-p h}^{s}$. Through Equ. (3) at each phonon mode and each phonon momentum, we have statistically derived (i) the $\lambda(\boldsymbol{q} ; v)_{\text {DNSSs }}$ associated with the electronic DNSSs by counting the decomposed ECFs over all electronic $k$ momenta on the locations of the DNSSs, $(i i)$ the $\lambda(\boldsymbol{q} ; v)$ over all the electron $k$ momenta in the surface BZ, and (iii) their ratio of $w=\frac{\lambda(q ; v) \text { DNSSs }}{\lambda(q ; v)}$. Among all the vibration $v$ modes and the phonon $\boldsymbol{q}$ momenta (see supplementary Table S1 and Table S2 [83]), the lowest and highest ratios are $w=77.6 \%$ and $w=84.5 \%$ originated from their $e$-ph couplings with the No.45 and No.3 phonon modes at $\boldsymbol{q}=(1 / 3,1 / 3,0)$ [85] in Fig. 3(a), respectively. Of course, the surface $e$-ph coupling strength $\lambda_{\mathrm{DNSSs}}$ over all phonon momenta and all vibration modes can be calculated by counting the ECFs at each $k$ momentum where the DNSSs appear through supplementary Equs. (S1 and S2) [83], consistently revealing that the DNSSs coupled $\lambda_{\text {DNSSs }}$ is $80.2 \%$ of the total $\lambda_{e-p h}^{s}$. As a result, these calculations confirm that the anomalously large $e$-ph coupling strength on the $\mathrm{Be}(0001)$ surface stems from the DNSSs in coupling with phonon states.

Theoretically, it is still possible to predict the superconducting $T_{c}$ of the $\mathrm{Be}(0001)$ surface with the theoretically derived $\lambda_{e-p h}^{s}=0.947$ in combining with the ECF of $\alpha^{2} F(\omega)$ in terms of the Dynes modified McMillan formula [86, 87]. In a range of the effective screened Coulomb repulsion constant $\mu=0.10$ $\sim 0.15$, the superconducting $T_{c}$ of the Be (0001) surface can be further estimated to be $15.3 \mathrm{~K} \sim 20.5 \mathrm{~K}$. However, even down to $12 \mathrm{~K}$ the ARPES experiment revealed no gap of the superconductivity [67]. Yet, this superconducting transition still needs to be confirmed, but it would strictly depend on the 
high-quality samples of Be (0001) surface.

In summary, we have evidenced that the topologically protected DNL-induced DNSSs lay a solid foundation for the novel enhancement of the surface $e$-ph coupling strength on the Be (0001) surface. It would highlight a potential application correlated with the $e$-ph coupling interaction for various topological Dirac nodal line semimetals.

Acknowledgments The work was supported by the $\mathrm{Na}$ - tional Science Fund for Distinguished Young Scholars (No. 51725103), by the National Natural Science Foundation of China (Grant Nos. 51671193 and 51474202), and by the Science Challenging Project No. TZ2016004. All calculations have been performed on the high-performance computational cluster in the Shenyang National University Science and Technology Park.

R. H. Li, and J. X. Li. contributed equally to this work.
[1] S. Ryu, and Y. Hatsugai, Phys. Rev. Lett. 89, 077002 (2002).

[2] T. T. Heikkilä, G. E. Volovik, JETP Lett. 93, 59 (2011).

[3] A. A. Burkov, M. D. Hook, and L. Balents, Phys. Rev. B 84, 235126 (2011).

[4] H. M. Weng, Y. Y. Liang, Q. N. Xu, R. Yu, Z. Fang, X. Dai, and Y. Kawazoe, Phys. Rev. B, 92, 045108 (2015).

[5] R. H. Li, H. Ma, X. Y, Cheng, S. L .Wang, D. Z. Li, Z. Z. Yu, Y. Y. Li and X.-Q. Chen, Phys. Rev. Lett. 117, 096401 (2016).

[6] R. Yu, H. M. Weng, Z. Fang, X. Dai, and X. Hu, Phys. Rev. Lett. 115, 036807 (2015).

[7] Y. Kim, B. J. Wieder, C. L. Kane, and A. M. Rappe, Phys. Rev. Lett. 115, 036806 (2015).

[8] L. S. Xie, L. M. Schoop, E. M. Seibel, Q. D. Gibson, W. W. Xie, and R. J. Cava, APL Materials 3, 083602 (2015).

[9] M. G. Zeng, C. Fang, G. Q. Chang, Y.-A. Chen, T. Hsieh, A. Bansil, H. Lin, and L. Fu, arXiv: 1504.3492 (2015).

[10] L. Lu, L. Fu, J. D. Joannopoulos, and M. Soljačić, Nature Photonics 7, 294-299 (2013).

[11] K. Mullen, B. Uchoa, and D. T. Glatzhofer, Phys. Rev. Lett. 115, 026403 (2015).

[12] Y. P. Du, F. Tang, D. Wang, L. Sheng, E.-J. Kan, C.-G. Duan, S. Y. Savrasov, and X. G. Wan, npj Quantum Materials 2, $2397-$ 4648 (2017).

[13] S.-Y. Yang, H. Yang, E. Derunova, S. S. P. Parkin, B. H. Yan, and M. N. Ali, Advances in Physics: X 3 1(2018).

[14] Q. N. Xu, R. Yu, Z. Fang, X. Dai, and H. M. Weng, Phys. Rev. B 95, 045136 (2017).

[15] H. Q. Huang, J. P. Liu, D. Vanderbilt, and W. H. Duan, Phys. Rev. B 93, 201114(R) (2016).

[16] B. J. Feng, B. T. Fu, S. Kasamatsu, S. Ito, P. Cheng, C.-C. Liu, Y. Feng, S. L. Wu, S. K. Mahatha, P. Sheverdyaeva, P. Moras, M. Arita, O. Sugino, T. C. Chiang, K. Shimada, K. Miyamoto, T. Okuda, K. H. Wu, L. Chen, Y. G. Yao, and I. Matsuda, Nature Commun. 8, 1007 (2017).

[17] J.-L. Lu, W. Luo, X.-Y. Li, S.-Q. Yang, J.-X. Cao, X.-G. Gong and H.-J. Xiang, Chinese Physics Letters 34, 057302 (2017).

[18] L. M. Schoop, M. N. Ali, C. Straßer, A. Topp, A. Varykhalov, D. Marchenko, V. Duppel, S. S. P. Parkin, B. V. Lotsch, and C. R. Ast, Nat. Commun. 7, 1196 (2016).

[19] J. Hu, Z. Tang, J. Liu, X. Liu, Y. Zhu, D. Graf, K. Myhro, S. Tran, C. N. Lau, J. Wei, and Z. Mao, Phys. Rev. Lett. 117, 016602 (2016).

[20] C. Fang, H. M. Weng, X. Dai, Z. Fang, Chinese Physics B 25, 117106 (2016).

[21] T. Hyart, R. Ojajärvi, and T. T. Heikkilä, J. Low Temp. Phys. 191, 35 (2018).

[22] H. Shapourian, Y. X.Wang, and S. Ryu, Phys. Rev. B 97, 094508 (2018).

[23] N. B. Kopnin, T. T. Heikkilä, and G. E. Volovik, Phys. Rev. B 83, 220503(R) (2011).
[24] G. E. Volovik, J. Supercond. Nov. Magn. 26,2887 (2013).

[25] Z. B. Yan, P. W. Huang, and Z. Wang, Phys. Rev. B 93, 085138 (2016).

[26] W. B. Rui, Y. X. Zhao, and Andreas P. Schnyder, Topological transport in Dirac nodal-line semimetals, Phys. Rev. B 97, 161113(R) (2018).

[27] J. Zhang, M. Gao, J. Zhang, X. Wang, X. Zhang, M. Zhang, W. Niu, and R. Zhang, Front. Phys. 13, 137201 (2018).

[28] R. Sankar, G. Peramaiyan, I. P. Muthuselvam, C. J. Butler, K. Dimitri, M. Neupane, G. Narsinga Rao, M. T. Lin ,and F. C. Chou, Sci. Rep. 7, 40603 (2017).

[29] M. B. Schilling, L. M. Schoop, B. V. Lotsch, M. Dressel, and A. V. Pronin, Phys. Rev. Lett. 119, 187401 (2017).

[30] S. P. Mukherjee and J. P. Carbotte, Phys. Rev. B 95, 214203 (2017).

[31] S. Ahn, E. J. Mele, and H. Min, Phys. Rev. Lett. 119, 147402 (2017).

[32] J. P. Carbotte, J. Phys.: Condens. Matter 29, 4 (2017).

[33] S. Pezzini, M. R. van Delft, L. M. Schoop, B. V. Lotsch, A. Carrington, M. I. Katsnelson, N. E. Hussey, and S. Wiedmann, Nature Physics 14, 178-183 (2018).

[34] L. Gao, J. T. Sun, J. C. Lu, H. Li, K. Qian, S. Zhang, Y. Y. Zhang, T. Qian, H. Ding, X. Lin, S. X. Du, and H. J. Gao, Adv. Mater. 30, 1707055 (2018).

[35] S. C. Li, Z. P. Guo, D. Z. Fu, X.-C. Pan, J. H. Wang, W.G. Ke, K.J. Ran, S. Bao, Z. Ma, Z.W. Cai, R.Wang, R. Yu, J. Sun, F.Q. Song, J.S. Wen, Science Bulletin, 63(9), 535-541 (2018).

[36] H. Zhang, Y. Xie, Z. Zhang, C. Zhong, Y. Li, Z. Chen, and Y. P. Chen, J. Phys. Chem. Lett. 8(8), 1707-1713 (2017).

[37] M. Hirayama, R. Okugawa, T. Miyake, and S. Murakami, Nature Commun. 8, 14022 (2017)

[38] J. X. Li, H. Ma, Q. Xie, S. B. Feng, S. Ullah, R. H. Li, J. H. Dong, D. Z. Li, Y. Y. Li, and X.-Q. Chen, Sci. China Mater. 61, 23-29, (2018).

[39] H. M. Weng, Y. Y. Liang, Q. N. Xu, R. Yu, Z. Fang, X. Dai, and Y. Kawazoe, Phys. Rev. B 92, 045108 (2015).

[40] R. Yu, H. M. Weng, Z. Fang, X. Dai, and X. Hu, Phys. Rev. Lett. 115, 036807 (2015).

[41] Y. Kim, B. J. Wieder, C. L. Kane, and A. M. Rappe, Phys. Rev. Lett. 115, 036806 (2015).

[42] L. S. Xie, L. M. Schoop, E. M. Seibel, Q. D. Gibson, W. W. Xie, and R. J. Cavaa, APL Mater. 3, 083602 (2015).

[43] K. Mullen, B. Uchoa, and D. T. Glatzhofer, Phys. Rev. Lett. 115, 026403 (2015).

[44] T. Bzdušek, Q. S. Wu, A. Rüegg, M. Sigrist, and A. A. Soluyanov, Nature 538, 75 (2016)

[45] R. Yu, Q. S. Wu, Z. Fang, and H. M. Weng, Phys. Rev. Lett. 119, 036401 (2017).

[46] J.-T. Wang, S.M. Nie, H.M. Weng, Y. Kawazoe, and C.F. Chen, Phys. Rev. Lett. 120, 026402 (2018). 
[47] J. X. Li, Q. Xie, S. Ullah, R. H. Li, H. Ma, D. Z. Li, Y. Y. Li, and X.-Q. Chen, Phys. Rev B 97, 054305 (2018)

[48] G. Q. Chang, S.-Y. Xu, X. T. Zhou, S.-M. Huang, B. Singh, B. K. Wang, I. Belopolski, J. X. Yin, S. T. Zhang, and A. Bansil, H. Lin, and M. Z. Hasan, Phys. Rev. Lett. 119, 156401 (2017).

[49] R. Bi, Z. B. Yan, L. Lu, and Z. Wang, Phys. Rev. B 96, 201305(R) (2017).

[50] X. Feng, Q. S. Wu, Y. Cheng, B. Wen, Q. Wang, Y. Kawazoe, p. Jena, Carbon 127, 527 (2018).

[51] Y. Sun, Y. Zhang, C.-X. Liu, C. Felser, and B. H. Yan, Phys. Rev. B 95, 235104 (2017).

[52] Y. Wu, L.-L. Wang, E. Mun, D. D. Johnson, D. X. Mou, L. N. Huang, Y. B. Lee, S. L. Bud'ko, P. C. Canfield, and A. Kaminski, Nat. Phys. 12, 667-671 (2016).

[53] G. Bian, T.-R. Chang, H. Zheng, S. Velury, S.-Y. Xu, T. Neupert, C.-K. Chiu, S.-M. Huang, D. S. Sanchez, I. Belopolski, N. Alidoust, P.-J. Chen, G.Q. Chang, A. Bansil, H.-T. Jeng, H. Lin, and M. Z. Hasan Phys. Rev. B 93, 121113 (2016).

[54] G. Bian, T.-R. Chang, R. Sankar, S.-Y. Xu, H. Zheng, T. Neupert, C.-K. Chiu, S.-M. Huang, G. Q. Chang, I. Belopolski, D. S. Sanchez, M. Neupane, N. Alidoust, C. Liu, B.K. Wang, C.-C. Lee, H.-T. Jeng, C.L. Zhang, Z.J. Yuan, S. Jia, A. Bansil, F.C. Chou, H. Lin and M. Z. Hasan Nat. Commun. 7, 10556 (2016).

[55] M. Neupane, I. Belopolski, M. M. Hosen, D. S. Sanchez, R. Sankar, M. Szlawska, S.-Y. Xu, K. Dimitri, N. Dhakal, P. Maldonado, P. M. Oppeneer, D. Kaczorowski, F.C. Chou, M. Z. Hasan, and T. Durakiewicz Phys. Rev. B 93, 201104 (2016).

[56] J.-W. Rhim, and Y. B. Kim, Phys. Rev. B 92, 045126 (2015).

[57] Y. Huh, E.-G. Moon, and Y. B. Kim, Phys. Rev. B 93, 035138 (2016).

[58] E. W. Plummer, and J. B. Hannon, Progress in Surface Science 46, 149-158 (1994).

[59] U. O. Karlsson, S. A. Flodstrom, R. Engelhardt, W. Gadeke, and E. E. Koch, Solid State Commun. 49, 711-714 (1984).

[60] I. Vobornik, J. Fujii, M. Mulazzi, G. Panaccione, M. Hochstrasser, and G. Rossi, Phys. Rev. B 72, 165424 (2005).

[61] R. A. Bartynski, E. Jensen, T. Gustafasson, and E. W. Plummer, Phys. Rev. B 32, 1921-1926 (1985).

[62] P. T. Sprunger, L. Petersen, E. W. Pulmmer, E. Laegsgaard, and F. Besenbacher, Science 275, 1764-1767 (1997).

[63] H. L. Davis, J. B. Hannon, K. B. Ray, and E. W. Plummer, Phys. Rev. Lett. 68, 2632-2635 (1992).

[64] L. I. Johansson, H. I. P. Johansson, J. N. Andersen, E. Lundgren, and R. Nyholm, Phys. Rev. Lett. 71, 2453-2456 (1993).

[65] P. J. Feibelman, Phys. Rev. B 46, 2532-2539 (1992).

[66] T. Balasubramanian, E. Jensen, X. L. Wu, and S. L. Hulbert, Phys. Rev. B 57, R6866 (1998).

[67] M. Hengsberger, D. Purdie, P. Segovia, M. Garnier, and Y. Baer, Phys. Rev. Lett. 83, 592-595 (1999).

[68] S.-J. Tang, J. Shi, B. Wu, P. T. Sprunger, W. L. Wang, V. Brouet,
X. J. Zhou, Z. Hussain, Z.-X. Shen, Z. Zhang, and E. W. Plummer, Phys. Stat. Sol. (b) 241, 2345-2352 (2004).

[69] G. Grimvall, The Electron-Phonon Interaction in Metals (NorthHolland, New York, 1981).

[70] G. D. Mahan and E. W. Plummer, in Electronic structure edited by K. Hohn and M. Scheffler, Handbook of Surface Science, Vol.2 (Elsevier, Amsterdan, 2000), Chap. 14, pp. 953-987.

[71] S. LaShell, E. Jensen, and T. Balasubramanian, Phys. Rev. B 61, 2371 (2000)

[72] A. Eiguren, S. de Gironcoli, E. V. Chulkov, P. M. Echenique, and E. Tosatti, Phys. Rev. Lett. 91, 166803 (2003).

[73] I. Y. Sklyadneva, E. V. Chulkov, P. M. Echenique, A. Eiguren, Surface Science, 600, 3792 (2006).

[74] M. Lazzeri and S. D. Gironcoli, Surf. Sci. 402“C404, 715 (1998).

[75] J. B. Hannon, E.W. Plumme, Shear horizontal vibrations at the (0001) surface of beryllium, J. Electron.Spectrosc. Relat. Phenom. 64/65, 683(1993).

[76] Ph. Hofmann and E. W. Plummer, Surf. Sci. 377-379, 330 (1997).

[77] T. Y. Chien, E. D. L. Rienks, M. F. Jensen, P. Hofmann, and E. W. Plummer, Phys. Rev. B 80, 241416 (2009).

[78] T. Y. Chien, X. B. He, S.-K. Mo, M. Hashimoto, Z. Hussain, Z.-X. Shen and E. W. Plummer, Phys. Rev. B 92, 075133 (2015).

[79] P. Hohenberg and W, Kohn, Phys. Rev. 136, B864 (1964).

[80] W. Kohn and L. J. Sham, Phys. Rev. 140, A1133 (1965).

[81] P. Giannozzi, S. Baroni, N. Bonini, M. Calandra, R. Car, C. Cavazzoni, D. Ceresoli, G. L. Chiarotti, M. Cococcioni , and I. Dabo, J. Phys.: Condens. Matter 21, 395502 (2009).

[82] J.P. Perdew and A. Zunger, Phys. ReV. B 23, 5048 (1981).

[83] The supplementary materials contain $(i)$ the computational methods of the DFT and the equations (S1 and S2) to calculate both the Eliashberg coupling function (ECF), $\alpha^{2} F(\omega)$, and the $e$-ph coupling strength, $\lambda$, (ii) Figure S1 to compare the theoretically derived ECFs and $\lambda$ of both bulk phase and the (0001) surface of the pure berrylium metal, (iii) Table S1 to list all the phonon $\boldsymbol{q}$ momenta considered here and their multiplicities in the (0001) surface BZ, (iv) Table S2 to list all the theoretically derived $\lambda(\boldsymbol{q} ; v)_{\text {DNSSs }}, \lambda(\boldsymbol{q} ; v)$ and their ratio $w=\frac{\lambda(\boldsymbol{q} ; v)_{\text {DNSSs }}}{\lambda(\boldsymbol{q} ; v)},(v)$ Figure S2 to compile $(\boldsymbol{q} ; v)$-resoved $\lambda(\boldsymbol{q} ; v)_{\text {DNSSs }}$ and $\lambda(\boldsymbol{q} ; v)$, and (vi) Figure $\mathrm{S} 3$ to show the anisotropic $e$-ph coupling strength along both $\bar{\Gamma}-\bar{M}$ and $\bar{\Gamma}-\bar{K}$ directions.

[84] G. Grimvall. in The Electron-Phonon Interaction in Metals (edited by E. Wohlfarth), Selected Topics in Solid State Physics(North-Holland, New York, 1981).

[85] For both phonon $\boldsymbol{q}$ and electron $\boldsymbol{k}$ momenta their units are of reciprocal lattice vectors, otherwise unless explicitly stated.

[86] W. L. McMillan, Phys. Rev. 167, 331 (1968).

[87] P. B. Allen and R. C. Dynes, Phys. Rev. B 12, 905 (1970). 